Lung cancer rarely involves the peritoneum without involvement of other metastatic sites. The most common regions of metastasis are the pleura, lung parenchyma, skeletal system, liver, brain, and adrenal glands. Compared with pleural metastasis, peritoneal involvement is rarely encountered in lung carcinoma. Ascites development is often described during late stages of the disease, following diagnosis in patients with serosal involvement other than pleural metastases. Diagnosis is based on histopathological or cytological examination confirmed by the presence of malign cells in the peritoneal fluids. Metastatic involvement of other areas is common and expected in cancer patients in whom peritoneal metastasis develop. There are no pulished reports of lung cancer cases with involvement of only the peritoneum without metastases to other more common regions. Herein we present a rare case of non-small cell lung carcinoma involving the peritoneum. The presented case had an unusual course and relapsed with peritoneal carcinomatosis without involvement of other regions.

Key words: non-small cell lung cancer; metastasis; peritoneal carcinomatosis.

\section{Relapse with isolated peritoneal metastasis in lung adenocarcinoma: case report and review of the literature}

\author{
Ozgur Tanriverdi', Sabri Barutca ${ }^{2}$, Nezih Meydan ${ }^{2}$
}

1Department of Medical Oncology, Mugla Sitki Kocman University Education and Research Hospital, Mugla, Turkey

2Adnan Menderes University, Faculty of Medicine, Department of Medical Oncology, Aydin, Turkey

\section{Introduction}

Lung cancer, which is among the most common tumors in adults, accounts for $12.4 \%$ of all new cancer cases worldwide, exhibiting one of the highest rates of mortality [1]. Of the patients with lung cancer, approximately $40 \%$ have metastases, presenting the main cause of mortality. The most common regions of metastasis are the pleura, lung parenchyma, skeletal system, liver, brain, and adrenal glands [1]. Metastasis to the peritoneum is rarely encountered during the clinical course of lung cancer, and there are limited treatment alternatives for such cases [1, 2].

Compared with pleural metastasis, peritoneal involvement is rarely encountered in lung carcinoma. In fact, peritoneal involvement may be asymptomatic and may only be determined through imaging techniques, although it may have a severe clinical course with high incidence of morbidity and mortality. Definite diagnosis of peritoneal involvement is confirmed by the presence of malignant cells in the peritoneal fluid [1, 2].

Metastatic involvement of other areas is common and expected in cancer patients in whom peritoneal metastases develop [1, 2]. To the best of our knowledge, there are no studies in the literature describing a lung cancer patient with a relapse in the peritoneal region but without metastases to distant and anticipated sites. Herein, we aimed to present a rare case of non-small cell lung carcinoma involving the peritoneum and to discuss, in light of the current limited literature, our patient who had an unusual course presenting with metastasis to the peritoneal surface without involvement of other regions.

\section{Case report}

A 59-year-old man presented to the chest diseases clinic of another hospital with fatigue, dyspnea, and hemoptysis, which began a few months earlier. Chest x-ray at that time showed increased density in the inferior right lung. Thoracic computed tomography (CT) confirmed that finding, based on observation of a $4 \times 3-\mathrm{cm}$ soft tissue mass that was obstructing the posterobasal segment and apical bronchus segment below the carina, an atelectatic area with pleural fluid, as well as multiple para-aortic and paratracheal lymphadenopathies (LAP) proximal to the carina, the largest of which was $1 \times 1 \mathrm{~cm}$. Bronchoscopic analysis showed blunting at the carina, mucosal tram-lines in both bronchi, and external compression of the right lung, particularly the lateral and posterior segments, but endobronchial lesions were not observed. Histopathological examination of transbronchial biopsy material showed adenocarcinoma, and then the patient was referred to our department.

The patient, a chemical engineer, did not have a history of any comorbidity other than diabetes mellitus for the past 5 years, which was managed 
with oral antidiabetics. As an ex-smoker for the last 10 years, the patient previously smoked 25 packs/year and did not use alcohol. He had no family history of cancer, and exposure to asbestosis was negative. His performance status was Eastern Cooperative Oncology Group (ECOG) 1, blood pressure was 130/85 mm Hg, and heart rate was 92 bpm and rhythmic. Physical examination did not show any pathological findings, except a coarse respiratory sound in the right hemithorax (posterior and lateral regions) and sporadic inspiratory rales. Serum lactate dehydrogenase level was 324 U/I (normal range: $125-243 \mathrm{U} / \mathrm{l})$, and other routine laboratory parameters were normal. Distal organ metastases were not noted with whole-body bone scintigraphy and abdominal and cranial CT. He was staged as T2N2MO (Stage IIIA).

The patient was administered neoadjuvant chemotherapy (28-day cycle) - cisplatin $80 \mathrm{mg} / \mathrm{m}^{2}$ (day 1 ) and gemcitabine $1000 \mathrm{mg} / \mathrm{m}^{2}$ (days 1, 8, and 15). According to the follow-up thoracic $C T$, the patient achieved a partial response following 2 cycles of the therapy, and then an additional cycle was administered. He was admitted to another hospital due to progressive shortness of breath, as well as heart palpitations present for the last five days. Physical examination revealed congested jugular, shortness of breath, orthopneic posture, and 1+ pitting edema in the bilateral pretibial areas. His arterial pressure was low $(90 / 60 \mathrm{~mm} \mathrm{Hg})$ and the heart beat rate was high (142/min). Prominent low voltage QRS complexes in all derivations were obtained on electrocardiogram. The patient underwent echocardiographic examination and pericardial effusion was observed. One hundred and fifty milliliters of bloody fluid were removed by echocardiography guided needle aspiration. Adenocarcinoma cells were noted based on cytological evaluation of the pericardial fluid (Fig. 1).

Positron emission tomography (PET)-CT for assessment showed a 36-mm hypermetabolic $\left(S_{U} U V_{\max }=4.8\right)$ lymph node in the subcarinal region that extended through the right intermediate bronchus posterior wall, a 51.6- $\mathrm{mm}$ mass density increase that resulted in the collapse of all peripheral basal

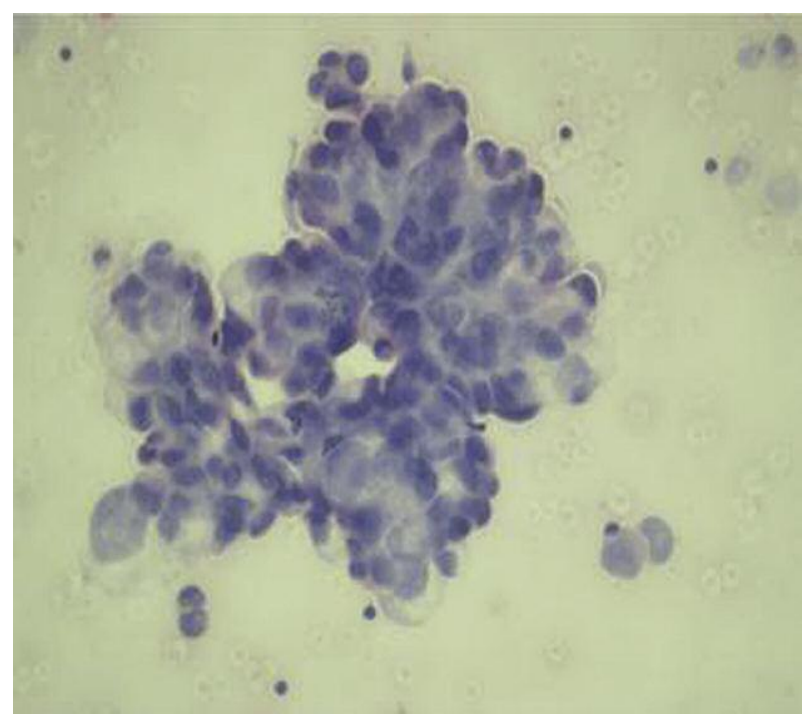

Fig. 1. Poorly differentiated carcinoma cells forming sporadic adenoid-like structures in pericardial fluid (HE, 200×) segments and increased glycolytic activity $\left(S U V_{\max }=5.9\right)$ in the internal structure of the suprahilar region peripheral to the basal segment bronchi, as well as the presence of minimal pericardial fluid. Clinically staged T4 (with malignant pericardial tamponade) N2M0, surgery was deemed inappropriate for the patient and he received 30-Gy palliative radiotherapy for the primary tumor and mediastinum. Response assessment after radiation treatment showed that there was a reduction in the mass formation around the right lung's inferior lobe apical segment and posterobasal segment bronchus. Lymph nodes noted in the carina during the previous analysis were not present; however, diffuse peritoneal fluid was a new finding. As such, cytological analysis of the paracentesis fluid was performed and poorly differentiated carcinoma cells forming sporadic adenoid-like structures were noted (Fig. 2). The serum acid-albumin fraction was 0.8. The patient did not have hepatic metastases, portal hypertension, or peripheral edema. Echocardiography did not demonstrate pericardial fluid or left ventricular systolic dysfunction. The patient was thought to have peritoneal carcinomatosis. The case relapsed with involvement of the peritoneum only, without metastases at other sites that are often involved in lung cancer, and a second opinion for diagnosis of malignant mesothelioma was requested from the pathology department. The patient's transbronchial biopsy specimen tumor cells immunohistochemically stained positive with cytokeratin 7 and thyroid transcription factor-1, but did not stain with cytokeratin 5/6. Tumor cells in pericardial and peritoneal fluid had similar morphology as the adenocarcinoma cells obtained via transbronchial biopsy. Malignant mesothelioma was ruled out based on these findings and the final diagnosis of primary lung adenocarcinoma was made. Then, second-line treatment (21-day cycle) with docetaxel ( $75 \mathrm{mg} / \mathrm{m}^{2}$ on day 1 ) was started. The patient could only receive 1 cycle of this chemotherapy regime and died.

\section{Discussion}

Although ascites can be seen at relapse in lung cancer, the incidence of isolated peritoneal carcinomatosis without

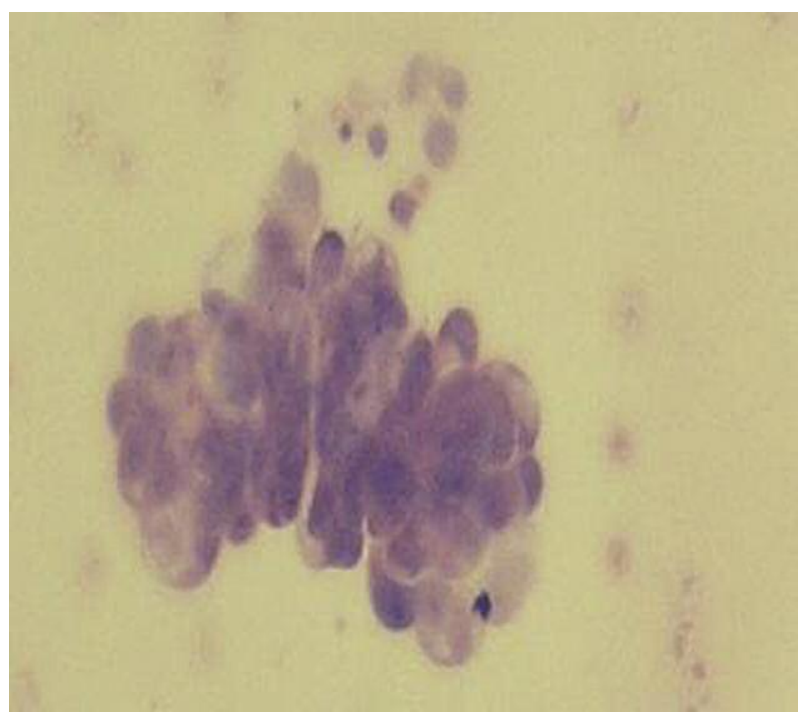

Fig. 2. Poorly differentiated carcinoma cells forming sporadic adenoid-like structures in ascites (HE, 200x) 
metastases in other parts of the body is very low [1, 2]. A search of the literature showed that only 1 case report and 2 individual case analyses regarding peritoneal involvement have been published $[1,3,4]$. There are no published reports of lung cancer cases with involvement of only the peritoneum without metastases to other more common regions $[2,5,6]$.

Although the frequency of peritoneal metastases in autopsy series is $2.7-16 \%$, in clinical studies it is 1-2\% [1]. Satoh et al. evaluated 1041 patients with lung cancer and reported that $1.2 \%(n=12)$ of the patients had clinically verified peritoneal carcinomatosis [4]. Peritoneal carcinomatosis has been reported in cases of non-small cell lung cancer. Peritoneal carcinomatosis was also reported relatively more frequently in association with adenocarcinoma subtypes [1]. Although small cell lung cancer is known to be the most aggressive subtype with the highest incidence of metastases, it is reported less frequently in peritoneal metastases series, probably due to characteristic occurrence of diffuse metastases at an early stage and short clinical duration. In their analysis of 30 patients with lung cancer and peritoneal metastases, Su et al. reported that 26 of the patients (86.6\%) had adenocarcinoma type, of which 20 were stage IV and 6 were stage IIIB. In that series only 3 patients had small cell subtype lung carcinoma, 1 had combined small cell/squamous cell lung carcinoma, and 1 had a pancreatic metastasis together with peritoneal carcinomatosis [1]. Based on explorative laparotomy, Sakar et al. described a patient with pancreatic metastasis and another with small cell lung carcinoma in the peritoneum that were studied for icterus [7].

Lung cancer can either spread locally and involve nearby tissues, such as the cavity outside the lungs (pleural space), or layers around the heart, or may travel to distant sites by vascular, lymphatic and transcoelomic routes/along serous membranes [1].

In patients with lung cancer signs and symptoms including abdominal distress, distension, and pain together with respiratory distress, as well as ileus, peripheral edema, nausea, and vomiting, were reported to be associated with peritoneal involvement. Su et al. reported that among their patients with peritoneal carcinomatosis $93.3 \%$ had abdominal distension, $23.3 \%$ had pretibial edema, $20 \%$ had constipation and ileus, $20 \%$ had abdominal pain and distress, and $6.7 \%$ had nausea/vomiting; however, metastases were diagnosed with CT performed to assess treatment response before clinical signs and symptoms were observed [1]. In the present case there was only abdominal discomfort at the time peritoneal carcinomatosis was diagnosed. No pronounced symptoms were noted with imaging techniques that were used to determine staging and response in the peritoneal region.

Ascites development is often described during late stages of the disease, following diagnosis in patients with serosal involvement other than pleural metastases. Su et al. noted ascites at baseline in only 4 of 30 patients, whereas they observed the development of peritoneal carcinomatosis after 253 days (range: $0-1156$ days) following diagnosis in other patients [1]. This period was 214 days in the present case, which is close to the previously reported mean.
It was reported that malign pleural fluid developed before the diagnosis of peritoneal carcinomatosis or malign pericardial effusion in $80 \%$ of patients with lung cancer and that distal organ involvement was also common [1, 2, 6]. In the present case, however, there were no findings of metastases in the bone, liver, brain, or other regions at the time peritoneal carcinomatosis was diagnosed. Given that peritoneal cytological examinations were positive during the clinical course, it would be reasonable to assume that the minimal pleural fluid shown by the baseline CT, which disappeared after chemotherapy, was most probably malignant.

Malignant mesothelioma is often associated with asbestos exposure. It is a tumor that originates from serosal surfaces such as the pleura, pericardium, peritoneum, and tunica vaginalis, and is frequently fatal [8]. In particular, differentiation between epithelioid malignant mesothelioma and primary lung adenocarcinoma that infiltrates the pleura or metastatic adenocarcinoma must be done. Due to the predetermined isolated serosal infiltration in the present case, malignant mesothelioma was included in the differential diagnosis. Unfortunately, recognizing epithelioid mesothelioma is difficult because it shares similar microscopic properties with adenocarcinoma. As such, immunohistochemical staining patterns were important in the differential diagnosis. An immunohistochemical technique that facilitates the diagnosis of epithelioid malignant mesothelioma by itself has not been reported $[8,9]$. Despite this, the most commonly accepted immunohistochemical markers for the diagnosis of mesothelioma are reported to be calretinin and cytokeratin 5/6 [9]. Ordonez reported cytokeratin 5/6-positive staining in all of their 60 malignant mesothelioma patients, but nonetheless only 1 of the 50 patients with adenocarcinoma stained positive with cytokeratin 5/6 [10]. King et al. reported that none of their 240 patients with malignant mesothelioma stained with TTF-1, while 281 of 366 (77\%) patients with lung adenocarcinoma stained positive with TTF-1 [11]. Nearly $25 \%$ of primary lung adenocarcinoma cases do not stain with TTF-1. Nonetheless, the existence of this immunohistochemical staining feature alone is regarded as sufficient to make a diagnosis of primary adenocarcinoma [9]. The present case did not have a history of asbestos exposure. Re-evaluation of the transbronchial biopsy specimen used for the initial diagnosis showed that there was positive staining for cytokeratin and TTF-1, whereas the tumor cells did not stain with cytokeratin $5 / 6$, which rules out a diagnosis of mesothelioma.

Median survival was reported as 2 months following peritoneal carcinomatosis in lung cancer patients [1, 2, 5]. Su et al. reported that median survival was 14.7 days in patients followed up with conservative therapy and 127.3 days in those who received aggressive treatment [1]. These data indicate a significant difference in survival between patients administered conservative and aggressive treatments. As other regions of metastases are also often involved in patients with peritoneal carcinomatosis, aggressive systemic treatment seems unfeasible, as performance scores are low in such patients. Four patients with adenocarcinoma and good performance scores were followed up with 4-week gefitinib treatment, and clinically good responses were achieved [1]. The second line option in the present case was docetaxel, as the 
patient used to be a smoker, gefitinib had not obtained approval for this type of use in our country, and other active chemotherapy options were still available.

In conclusion, peritoneal carcinomatosis in patients with lung cancer occurs quite infrequently and is usually accompanied by other systemic metastases. The case presented here is unusual, as the patient developed isolated peritoneal involvement while receiving treatment for peritoneal metastases. This case suggests that pleural metastases may respond better to systemic treatment than other serosal regions in lung adenocarcinoma.

The authors declare no conflict of interest.

\section{References}

1. Su HT, Tsai CM, Perng RP. Peritoneal carcinomatosis in lung cancer. Respirology 2008; 13: 465-7.

2. Quraishi MA, Costanzi JJ, Hokanson J. The natural history of lung cancer with pericardial metastases. Cancer 1983; 51: 740-2.

3. Ba O, Almeida F, Fondronnier C, Michalski M, Gossot D, Savinelli F, Le Maignan C, Misset JL, et al. Abdominal pain during the course of lung cancer. Rev Pneumol Clin 2007; 63: 100-3.

4. Satoh H, Ishikawa H, Yamashita YT, Kurishima K, Ohtsuka M, Sekizawa K. Peritoneal carcinomatosis in lung cancer patients. Oncol Rep 2001; 8: 1305-7.

5. Hoda RS, Cangiarella J, Koss LG. Metastatic squamous cell carcinoma in pericardial effusion: Report of four cases, two with cardiac tamponade. Diagn Cytopathol 1998; 18: 422-4

6. Zuo ZL Pericardial metastasis of lung cancer-report of 13 cases. Zhong Liu Za Zhi 1989; 11: 142-4.

7. Sakar A, Kara E, Aydede H, Ayhan S. A case of a small cell lung carcinoma presenting with jaundice due to pancreatic metastasis. Tuberk Toraks 2005; 53: 181-4.

8. Tang P, Vatsia SK, Teichberg S, Kahn E. Pulmonary adenocarcinoma stimulating malignant mesothelioma. Arch Pathol Lab Med 2001; 125: $1598-600$

9. Akyildiz EU, Oz B, Aki H, Demirkaya A. Immunohistochemistry for differentiation pleural epithelioid malignant mesothelioma from adenocarcinoma: a panel for selection. Turk Toraks Dergisi 2010 11: 144-8.

10. Ordonez NG. The immunohistochemical diagnosis of mesothelioma: a comparative study of epithelioid mesothelioma and lung adenocarcinoma. Am J Surg Pathol 2003; 27: 1031-51.

11. King J, Thatcher N, Pickering C, Hasleton P. Sensitivity and specifitcity of immunohistochemical antibodies used to distinguish between benign and malignant pleural disease: a systematic review of published reports. Histopathology 2006; 49: 561-8.

\section{Address for correspondence}

\section{Ozgur Tanriverdi}

Department of Medical Oncology

Mugla Sitki Kocman University Education and

Research Hospital

Mugla, Turkey

tel. +902564441256 (1091)

fax +902562131705

e-mail: ozgurtanriverdi@hotmail.com

Submitted: $\quad 23.05 .2011$

Accepted: $\quad 2.04 .2012$ 